\title{
Smoking as a Preventable Risk Factor for Rheumatoid Arthritis: Rationale for Smoking Cessation Treatment in Patients with Rheumatoid Arthritis
}

\author{
Sungwon Roh \\ Department of Psychiatry, Hanyang University College of Medicine, Seoul, Korea
}

Rheumatoid arthritis (RA) is a chronic inflammatory disease of multifactorial etiology. Smoking is considered one of the most established environmental risk factors for RA development and severity. A large proportion of patients with RA have a high prevalence of smoking history. Previous studies have provided evidence suggesting that smoking is associated with the development of RA. Smoking has been associated with several pathogenic mechanisms on RA development such as oxidative stress, inflammation, and epigenetic changes. There is a need for public health campaigns to educate the public regarding these risks and preventive measures that reduce smoking are essential and may result in a decline in RA incidence. Encouragement of smoking cessation is especially warranted in relatives of patients with RA. Recently, RA-specific smoking cessation interventions have been developed. This review will summarize the knowledge accumulated to date concerning associations between smoking and RA. (J Rheum Dis 2019;26:12-19)

Key Words. Smoking, Nicotine, Tobacco, Smoking cessation, Rheumatoid arthritis

\section{INTRODUCTION}

Rheumatoid arthritis (RA) is an autoimmune-mediated inflammatory disease that affects $0.5 \% \sim 1 \%$ of the overall population [1]. RA results from the interaction between genetic constitution and environmental triggers. The disease has been classified into two major subsets based on the presence or absence of anti-citrullinated protein antibodies (ACPA) [2-4].

Our understanding of the pathogenesis of RA has progressed over the past two decades thanks to epidemiologic and translational studies. Specifically, epidemiologic investigations of smoking and risk of RA have allowed the construction of a paradigm for RA development and generated novel hypotheses that have been tested in translational studies to further understand the biology of RA pathogenesis. Many environmental factors have been associated with an increased risk of developing
RA, but to date smoking is the only environmental risk factor that has been extensively studied and is widely accepted. The association of smoking with RA led investigators to initially consider the lung as a site of RA pathogenesis. Recent studies have revealed a link between smoking and inflammatory arthritis. Indeed, the particular association of smoking with RA seropositive for ACPA led investigators to consider citrullination as an essential biologic process in RA pathogenesis. The identification of abnormalities in lung structure and local autoantibody production further support this hypothesis [5]. Cigarette smoking has also been associated with inflammatory joint symptoms in unaffected first-degree relatives of RA patients [6]. Smoking may play an important role in different phases pathogenesis RA before the manifestation of clinical symptoms.

Smoking is associated with an increased risk of developing seropositive RA (rheumatoid factor $[\mathrm{RF}]$ and/or

Received : June 18, 2018, Revised : September 27, 2018, Accepted : October 15, 2018

Corresponding to : Sungwon Roh iD http://orcid.org/0000-0003-4557-3542

Department of Psychiatry, Hanyang University College of Medicine, 222 Wangsimni-ro, Seongdong-gu, Seoul 04763, Korea. E-mail : swroh@hanyang.ac.kr

Copyright (c) 2019 by The Korean College of Rheumatology. All rights reserved.

This is a Open Access article, which permits unrestricted non-commerical use, distribution, and reproduction in any medium, provided the original work is properly cited. 
ACPA positivity). Recent studies have shown that smoking can influence disease phenotype, with the development of more aggressive disease and more severe joint damage; but other studies have reported contradictory results. Recent data have also suggested that smokers respond less favorably to antirheumatic therapy [7]. This review will address smoking as a risk factor for RA, the controversies relative to the effects of tobacco on RA, the role of nicotine in RA pathogenesis, and the benefits of smoking cessation in patient with RA.

The available literature including meta-analysis, reviews, randomized controlled trials, systematic reviews, clinical trials, and case reports written in English and published within the past 10 years was reviewed. A bibliography review of the terms rheumatoid arthritis, smoking, nicotine, tobacco and smoking cessation was performed using the PubMed database. A total of 924 publications were identified, and following the assessment of each study, 64 articles were selected that were related with the main theme of this review. This study was exempted from Hanyang University Hospital Institutional Review Board evaluation in 2018.

\section{MAIN SUBJECTS}

\section{Smoking as a risk factor for rheumatoid arthritis}

The risk of developing RA in smokers is known to be double that of non-smokers. Previous epidemiological studies have identified smoking as an important risk factor for RA [4,8-15]. Numerous environmental factors have been associated with an increased risk of developing RA, but to date tobacco smoking has been the most important environmental risk factor to be extensively studied and widely accepted. Multiple studies have reported odds ratios (OR) of association between smoking and RA of 2 or greater, with estimates that exposure to smoking accounts for $20 \% \sim 30 \%$ of the environmental risk for RA [16]. Exposure to cigarette smoking was first linked to RA over 30 years ago [15]. Smoking is now recognized as the most established environmental risk factor for the development of RA [17]. Several studies have indicated that male smokers have a higher risk of developing RA than females $[10,12,14]$, whereas others have demonstrated that female smokers have a higher risk of RA $[11,13,15]$. A meta-analysis concluded that lifelong cigarette smoking was positively associated with the risk of RA even among smokers with a low lifelong exposure levels [9]. In addition, a few large-scale epidemiologic studies have al- so provided support for smoking being a stronger risk factor of RA in men than in women $[17,18]$; this might indicate that there are sex-related differences in the effects of smoking or that women have different risk factors for the development of RA.

Smoking is associated with ACPA-positive cases of RA [19]. ACPA-positivity correlates with disease activity. A previous study found that smoking is responsible for $35 \%$ of ACPA-positive cases in a dose-dependent manner (55\% in patients with 2 copies of shared epitope [SE] alleles) [20]. Several previously published studies have reported the association of the SE and smoking with anti-cyclic citrullinated peptide (CCP) negative as well as anti-CCP positive RA. In case-control Korean study, smoking was shown to be associated with anti-CCP-positive RA (OR 2.22) and anti-CCP-negative RA (OR 2.80). Smoking also increased RA susceptibility in individuals with SE alleles, regardless of their anti-CCP antibody [21]. In the absence of SE, smoking conferred risk for anti-CCP negative subsets [22]. Association of smoking with CCP-negative RA has also been reported in a Caucasian population.

The risk of RA in ex-smokers diminished with time. The risk of RA rose with increasing duration of smoking. Moreover, the risk of developing ACPA-positive RA diminished with time after cessation of smoking. Lifelong cigarette smoking was positively associated with the risk of RA even among smokers with a low lifelong exposure. The risk of RA did not further increase with an exposure higher than 20 pack-years [9].

Importantly, there are several features of the relationship between smoking and RA that may mediate the increased risk of developing RA. As indicated above, smoking is most strongly associated with ACPA-positive RA, and especially ACPA-positive RA in the setting of a SE [16]. Smoking is known as a risk factor of RA, especially in RF-positive RA men and heavy smokers [13]. Furthermore, smoking has long been associated with the presence of RF even in the absence of RA [23]. This suggests that there may be biological interactions between factors that drive RA development, or at the very least RA-related autoimmunity. As a relevant example, it has been proposed that smoking may lead to increased citrullination, which, within the context of the genetic background, may lead to increased levels of citrullinated proteins and the generation of ACPA, although other local and systemic effects of smoking may also influence immunity $[19,23,24]$. 
A recent Swedish population-based case-control study [25] demonstrated that smoking increases the risk of both subsets of RA with a more pronounced influence on the risk of ACPA-positive RA than in the ACPA-negative subset. For both subsets, there seemed to be a threshold (2.5 pack-years for ACPA-positive RA and 5 pack-years for ACPA-negative RA) below which, no association between smoking and RA occurred. A dose-response association was observed between cumulative dose of smoking and risk of developing-ACPA positive RA. Thus, duration of smoking had a higher influence on the association between smoking and RA than did the intensity of smoking. The Epidemiological Investigation of Rheumatoid Arthritis (EIRA) study from Sweden found that the interaction between smoking and silica exposure regarding ACPA-positive RA among males depended on the cumulative dose of smoking. The additive interaction effect between these two exposures might require over 10 years to disappear [26].

Furthermore, because smoking has also been associated with increased disease activity, its actions may go beyond the initiation of RA [27]. Thus, a major unanswered question regarding the role of smoking in RA is where it acts in the natural history of RA. Specifically, does exposure to cigarette smoking act to trigger the initial autoimmunity or does it drive the propagation of autoimmunity to the point of disease? Data from twin studies in Sweden have suggested that smoking possibly affects after the initial generation of RA-related autoimmunity and may be related to prolonged "high-dose/intensity" smoking, as measured by pack-years, although other studies suggest that it is more the duration of smoking rather than intensity that imparts risk for RA $[28,29]$.

\section{Pathogenic effects of smoking on rheumatoid ar- thritis}

Although the exact pathogenic effects of smoking on RA still remain uncertain, several mechanisms have been proposed to better understand how tobacco smoking plays a role in RA [7,30,31]. Smoking can increase oxidative stress, which increases in rheumatoid inflammation due to impairment of antioxidative mechanisms caused by free radicals contained in tar and vapor of smoke and that have been implicated in the etiology of RA [32,33]. Smoking acts on both cellular and humoral aspects of the immune system to cause a systemic proinflammatory state [34]. The effects of tobacco smoking on immune system are to trigger morphological, physiological, bio- chemical, and enzymatic changes that lead to impaired inflammatory responses [35]. Smoking is known to increase levels of proinflammatory cytokines, interleukin (IL)-17, which is an important contributor to RA pathogenesis and chronicity [36]. Epigenetic changes including DNA methylation has been explored as these may play an important role in gene regulation and development of RA [37]. Tobacco smoking has been reported to lead to extensive genome-wide changes in DNA methylation [38].

There are also systemic effects of smoking, and it is possible that these lead to changes within joints that drive RA. In this regard, in a study of first-degree relatives of patients with RA, joint tenderness and swelling were associated with smoking even in the absence of RA-related autoantibodies, raising the possibility that smoking may have early direct joint effects that could be related to the future development of RA [6]. In addition to baseline erosion, erythrocyte sedimentation rate, and C-reactive protein, current smoking was identified as a strong independent risk factor (adjusted OR=2.17, 95\% CI 1.06 4.45) for radiographic progression in early RA [39].

As discussed above, citrullination has been reported to be an important factor for the development of RA in the ACPA-positive subset. An increasing body of evidence has linked chronic inflammatory events in the lungs of smokers to the production of ACPAs and development of RA [40]. Previous meta-analysis suggested a gene-environmental interaction between smoking and SE for the development of ACPA [41].

There is a gene-environment interaction between smoking and the HLA-DRB1 SE genotype in seropositive RA patients [21,42]. Smoking interaction with HLA-DRB1 risk alleles increase the specificity, magnitude and diversity of the ACPA response (such as citrullinated $\alpha$-enolase, fibrinogen, and vimentin peptides, etc., not only CCP). A meta-analysis indicated that both smoking and protein tyrosine phosphatase non-receptor 22 (PTPN22) risk allele were associated with ACPA positivity [43].

\section{Anti-inflammatory effect of nicotine: benefits of nicotine on rheumatoid arthritis}

Paradoxically, nicotine has been reported to reduce inflammation, that is, nicotine reduced joint swelling, pain, and bone destruction, and alleviated synovial inflammation through the activation of the cholinergic pathway in a mouse model of RA [44]. Furthermore, nicotine attenuated tumor necrosis factor (TNF)- $\alpha$ induced IL-6 and IL-8 release in fibroblast-like synoviocytes from 
RA patients [45]. These results support the important immunosuppressive function of nicotine in tobacco smoke. A large prospective French cohort of patients with early RA revealed smoking status had no significant effect on RA disease activity and disability, but did reduce 1-year radiographic disease progression [46]. The anti-inflammatory role of nicotine may explain the lower systemic inflammation and structural disease progression in current smokers with early RA. A Swedish epidemiological study showed that the use of smokeless tobacco (moist snuff) was not associated with the risk of either ACPA-positive or ACPA-negative RA. The increased risk of RA associated with smoking was most likely not due to nicotine [47]. This means that different inhaled constituents of tobacco smoke other than nicotine may be more likely be involved in the pathogenesis of ACPA-positive RA.

Conversely, a recent study explored the effects of nicotine on neutrophil extracellular trap (NET) formation [48]. In this study, using neutrophils of RA patients and collagen-induced mice, the authors demonstrated that nicotine increases NETosis, which leads to increasing levels of NETs and may play a crucial role in accelerating arthritis.

A previous multi-cohort study indicated that the effects of smoking on joint damage were mediated via ACPA and that smoking is not an independent risk factor for radiological progression in RA [49]. In addition, no association was identified between second-hand exposure to tobacco smoke and disease activity in RA [50]. A Swedish cohort study showed that moist snuff was not associated with RA, whereas tobacco smoke was related to an increased risk for RA [51]. In other words, smokeless tobacco does not increase the risk of RA, suggesting that inhaled non-nicotinic components of cigarette smoke are more important than nicotine itself in the etiology of RA.

Of patients enrolled in a large randomized controlled trial of early RA with poor prognostic factors, smoking status did not impact on treatment responses in those receiving early combination or initial methotrexate with step-up therapy at 24 weeks if the disease was still active [52]. Given that smoking may not be a risk factor for the perpetuation of disease activity or progression, smoking cessation cannot be recommended to prevent severe outcome in early RA patients [46]. American and Swedish longitudinal observational studies have reported that smoking cessation after the onset of RA did not have an impact on the poor prognosis of smokers with RA
$[53,54]$. However, these results certainly cannot be used to advocate smoking in RA. All RA patients should be encouraged to stop smoking.

\section{Benefits of smoking cessation on disease activity of rheumatoid arthritis}

A Swedish cohort study indicated that the risk of RA decreased over time following smoking cessation, nevertheless, when compared to never smokers the risk was still statistically significantly higher [55]. The Swedish EIRA study showed compelling evidence recommending that those with a family history of RA should stop smoking [20]. This study demonstrated that smoking cessation reduced the risk of developing ACPA-positive RA. The effect of smoking cessation in individuals previously diagnosed with RA is unknown. Encouragement of smoking cessation is especially warranted in relatives of patients with RA [56].

Despite the success of TNF-alpha inhibitor (TNFi) treatment in RA, a substantial number of patients necessitate discontinuation. TNFi discontinuation is predicted by current smoking and by the number of previously used biological disease-modifying anti-rheumatic drugs (DMARDs), as well as by pack-years of smoking [57].

As discussed above, the relationship between smoking, ACPA and RA has been demonstrated by several studies and reports. Therefore, it is essential to inform patients of the hazardous role of smoking in the development and progression of RA. Moreover, as autoimmune diseases in general cause accelerated atherosclerosis due to constant inflammation, and increase the risk of cardiovascular disease, it is imperative that patients understand that smoking cessation is as important as therapy with DMARDs or biologics to achieve remission and improve quality of life [58].

For both ACPA-positive and negative subsets of RA, the detrimental effects of smoking decrease after smoking cessation. Twenty years after smoking cessation, there was no longer an association between smoking and risk of ACPA-negative RA, whereas the association between smoking and ACPA-positive RA risk persisted and was dependent on the cumulative dose of smoking [25].

\section{Clinical practices for smoking cessation in patients with rheumatoid arthritis}

An international survey of clinical practices regarding smoking cessation revealed that patient recommendations for smoking cessation within rheumatology departments 
were not homogeneous [59]. These data highlight the need to improve smoking cessation recommendations for patients with RA. The first interventional study in smokers with RA showed that a score of less dependence and previous attempts to quit smoking were significantly associated with definitive smoking cessation at 12 months [60]. The intervention consisted of the following: (1) a baseline visit, which included verbal and written advice by the rheumatologist, emphasizing the practical benefits of smoking cessation; (2) a follow-up visit to the nurse after 3 months for reinforcement and to receive pharmacological treatment to help patients quit smoking. Smoking cessation in RA may lead to reduce the burden of comorbidities. A RA-patient-specific smoking cessation intervention was developed, matching support to specific issues within an individual patient's experience [61]. However, the lack of added benefit of the tailored intervention suggested that brief advice and nicotine replacement therapy (NRT) are currently the best practice for supporting people with RA who wish to quit smoking [62]. This novel RA-specific smoking cessation intervention had 2 very important components, that is, individualized support and recommendations received from the educators [63]. Both components were considered pivotal to the success of the intervention. Although these novel psychosocial intervention approaches provide a variety of widely accepted and useful components, individual participants need to be motivated to give up smoking.

Smokers with RA may have different motivations for, and barriers to, quitting. There are physical limitations and disease-associated factors that may adversely affect smoking cessation in RA patients. Five key barriers to smoking cessation faced by RA patients have been identified in a previous study [64]. First, patients were unaware of the relationship between smoking and RA and therefore did not perceive this as a reason to quit. Second, smoking was used as a distraction from pain. Third, patients found it difficult to exercise and therefore were unable to use exercise as an alternative distraction. Fourth, smoking was used as a coping mechanism for the frustrations of living with RA. Fifth, patients felt unsupported and isolated from other RA non-smoking patients. These barriers and targeted interventions for patients with RA are outlined in detail in Table 1. Moreover, becoming aware of the effects of smoking on arthritis may represent an important motivation to quit smoking and may counter RA-specific barriers to smoking cessation [65].
A recent ongoing randomized controlled trial protocol will examine whether intensive smoking cessation intervention may help smokers with RA to achieve continuous smoking cessation and, secondly, to reduce RA disease activity [66]. The intervention includes individual motivational counselling in combination with tailored NRT. In the motivational counselling sessions, a smoking cessation counsellor's role is to ask, listen and follow the participants cue and to adapt formal information to the participant's motivational stage. Five sessions contain different themes. The first meeting is an introduction to the counselling course and preparation for smoking cessation, including the participant's smoking status and their motivation for cessation. The second meeting aims to prepare the participant for the three first days without smoking. The third meeting aims to help the participant with issues concerning quitting smoking, including risk situations, relapse, reward and social network, and smoking cessation. The fourth meeting includes maintaining motivation, physical activity, handling of stress and mood swings. The fifth (final) meeting includes continuing help with smoking cessation and preparation for the time after

Table 1. Barriers to smoking cessation and targeted interventions in RA

\begin{tabular}{|c|c|}
\hline Barrier & Intervention \\
\hline $\begin{array}{l}\text { 1. Limited knowledge of } \\
\text { the relationship between } \\
\text { smoking and RA }\end{array}$ & $\begin{array}{l}\text { Education about the links } \\
\text { between smoking and RA }\end{array}$ \\
\hline 2. Uncontrolled pain & Managing RA pain \\
\hline \multirow[t]{5}{*}{ 3. Inability to exercise } & Hand exercise for RA \\
\hline & General exercise for RA \\
\hline & Community exercise for RA \\
\hline & Hydrotherapy \\
\hline & $\begin{array}{l}\text { Physiotherapist or occupatioal } \\
\text { therapist referral }\end{array}$ \\
\hline \multirow{3}{*}{$\begin{array}{l}\text { 4. Using smoking as a } \\
\text { coping strategy }\end{array}$} & Identifying smoking triggers \\
\hline & $\begin{array}{l}\text { Discussing available support } \\
\text { (social, psychological and } \\
\text { physical) }\end{array}$ \\
\hline & Using NRT \\
\hline \multirow[t]{2}{*}{ 5. Lack of support } & $\begin{array}{l}\text { Suitable support for smoking } \\
\text { cessation }\end{array}$ \\
\hline & $\begin{array}{l}\text { Advice from arthritis } \\
\text { educatior: pain, } \\
\text { complementary therapies, } \\
\text { medications, pacing, } \\
\text { fatigue, sleep, etc. }\end{array}$ \\
\hline
\end{tabular}

RA: rheumatoid arthritis, NRT: nicotine replacement therapy. 
the intervention. The NRT is tailored individually according to the Fagerström Test for Nicotine Dependence [67]. Participants are able to choose between the NRT products, including a patch, chewing gum, inhalator or mouth spray. The participants note their tobacco and nicotine replacement consumption in a smoking diary.

\section{CONCLUSION}

Taken together, most studies have highlighted the risk of RA associated with smoking. Chronic inflammatory mechanisms active in the lungs of smokers lead to the production of ACPA, which, in turn, drive the development of RA. These mechanistic insights not only reinforce the association between smoking and risk of RA, but also the necessity to increase the level of awareness of those at highest risk. Smoking is one of the most prevalent modifiable risk factors for RA. Previous studies have highlighted that smoking cessation may reduce, though not remove, the risk of RA in women. The clearly increased risk of RA development even among former smokers is another reason to persuade women not to start smoking. Creation of awareness of the associated risks, assessment of smoking status, and implementation of smoking cessation treatment alternatives must be included in the routine clinical management of patients presenting with suspected RA.

\section{CONFLICT OF INTEREST}

No potential conflict of interest relevant to this article was reported.

\section{REFERENCES}

1. Smolen JS, Aletaha D, McInnes IB. Rheumatoid arthritis. Lancet 2016;388:2023-38.

2. Avouac J, Gossec L, Dougados M. Diagnostic and predictive value of anti-cyclic citrullinated protein antibodies in rheumatoid arthritis: a systematic literature review. Ann Rheum Dis 2006;65:845-51.

3. Huizinga TW, Amos CI, van der Helm-van Mil AH, Chen W, van Gaalen FA, Jawaheer D, et al. Refining the complex rheumatoid arthritis phenotype based on specificity of the HLA-DRB1 shared epitope for antibodies to citrullinated proteins. Arthritis Rheum 2005;52:3433-8.

4. Padyukov L, Silva C, Stolt P, Alfredsson L, Klareskog L. A gene-environment interaction between smoking and shared epitope genes in HLA-DR provides a high risk of seropositive rheumatoid arthritis. Arthritis Rheum 2004;50: 3085-92.
5. Sparks JA, Karlson EW. The roles of cigarette smoking and the lung in the transitions between phases of preclinical rheumatoid arthritis. Curr Rheumatol Rep 2016;18:15.

6. Sparks JA, Chang SC, Deane KD, Gan RW, Kristen Demoruelle M, Feser ML, et al. Associations of smoking and age with inflammatory joint signs among unaffected first-degree relatives of rheumatoid arthritis patients: results from studies of the etiology of rheumatoid arthritis. Arthritis Rheumatol 2016;68:1828-38.

7. Ruiz-Esquide V, Sanmartí R. Tobacco and other environmental risk factors in rheumatoid arthritis. Reumatol Clin 2012;8:342-50.

8. Costenbader KH, Feskanich D, Mandl LA, Karlson EW. Smoking intensity, duration, and cessation, and the risk of rheumatoid arthritis in women. Am J Med 2006;119: 503.e1-9.

9. Di Giuseppe D, Discacciati A, Orsini N, Wolk A. Cigarette smoking and risk of rheumatoid arthritis: a dose-response meta-analysis. Arthritis Res Ther 2014;16:R61.

10. Heliövaara M, Aho K, Aromaa A, Knekt P, Reunanen A. Smoking and risk of rheumatoid arthritis. J Rheumatol 1993;20:1830-5.

11. Karlson EW, Lee IM, Cook NR, Manson JE, Buring JE, Hennekens $\mathrm{CH}$. A retrospective cohort study of cigarette smoking and risk of rheumatoid arthritis in female health professionals. Arthritis Rheum 1999;42:910-7.

12. Krishnan E, Sokka T, Hannonen P. Smoking-gender interaction and risk for rheumatoid arthritis. Arthritis Res Ther 2003;5:R158-62.

13. Sugiyama D, Nishimura K, Tamaki K, Tsuji G, Nakazawa T, Morinobu A, et al. Impact of smoking as a risk factor for developing rheumatoid arthritis: a meta-analysis of observational studies. Ann Rheum Dis 2010;69:70-81.

14. Uhlig T, Hagen KB, Kvien TK. Current tobacco smoking, formal education, and the risk of rheumatoid arthritis. J Rheumatol 1999;26:47-54.

15. Vessey MP, Villard-Mackintosh L, Yeates D. Oral contraceptives, cigarette smoking and other factors in relation to arthritis. Contraception 1987;35:457-64.

16. Klareskog L, Gregersen PK, Huizinga TW. Prevention of autoimmune rheumatic disease: state of the art and future perspectives. Ann Rheum Dis 2010;69:2062-6.

17. Stolt P, Bengtsson C, Nordmark B, Lindblad S, Lundberg I, Klareskog L, et al. Quantification of the influence of cigarette smoking on rheumatoid arthritis: results from a population based case-control study, using incident cases. Ann Rheum Dis 2003;62:835-41.

18. Kochi Y, Thabet MM, Suzuki A, Okada Y, Daha NA, Toes $\mathrm{RE}$, et al. PADI4 polymorphism predisposes male smokers to rheumatoid arthritis. Ann Rheum Dis 2011;70:512-5.

19. Klareskog L, Stolt P, Lundberg K, Källberg H, Bengtsson C, Grunewald J, et al. A new model for an etiology of rheumatoid arthritis: smoking may trigger HLA-DR (shared epitope)-restricted immune reactions to autoantigens modified by citrullination. Arthritis Rheum 2006;54:38-46.

20. Källberg H, Ding B, Padyukov L, Bengtsson C, Rönnelid J, Klareskog L, et al. Smoking is a major preventable risk factor for rheumatoid arthritis: estimations of risks after various exposures to cigarette smoke. Ann Rheum Dis 2011;70: 508-11.

21. Bang SY, Lee KH, Cho SK, Lee HS, Lee KW, Bae SC. 
Smoking increases rheumatoid arthritis susceptibility in individuals carrying the HLA-DRB1 shared epitope, regardless of rheumatoid factor or anti-cyclic citrullinated peptide antibody status. Arthritis Rheum 2010;62:369-77.

22. Fisher BA, Bang SY, Chowdhury M, Lee HS, Kim JH, Charles $\mathrm{P}$, et al. Smoking, the HLA-DRB1 shared epitope and ACPA fine-specificity in Koreans with rheumatoid arthritis: evidence for more than one pathogenic pathway linking smoking to disease. Ann Rheum Dis 2014;73:741-7.

23. Arnson Y, Shoenfeld Y, Amital H. Effects of tobacco smoke on immunity, inflammation and autoimmunity. J Autoimmun 2010;34:J258-65.

24. Johannsen A, Susin C, Gustafsson A. Smoking and inflammation: evidence for a synergistic role in chronic disease. Periodontol 2000 2014;64:111-26.

25. Hedström AK, Stawiarz L, Klareskog L, Alfredsson L. Smoking and susceptibility to rheumatoid arthritis in a Swedish population-based case-control study. Eur J Epidemiol 2018;33:415-23.

26. Zeng P, Chen Z, Klareskog L, Alfredsson L, Bengtsson C, Jiang X. Amount of smoking, duration of smoking cessation and their interaction with silica exposure in the risk of rheumatoid arthritis among males: results from the Swedish Epidemiological Investigation of Rheumatoid Arthritis (EIRA) study. Ann Rheum Dis 2018;77:1238-41.

27. Malm K, Bremander A, Arvidsson B, Andersson ML, Bergman S, Larsson I. The influence of lifestyle habits on quality of life in patients with established rheumatoid arthritis-a constant balancing between ideality and reality. Int J Qual Stud Health Well-being 2016;11:30534.

28. Hensvold AH, Magnusson PK, Joshua V, Hansson M, Israelsson L, Ferreira R, et al. Environmental and genetic factors in the development of anticitrullinated protein antibodies (ACPAs) and ACPA-positive rheumatoid arthritis: an epidemiological investigation in twins. Ann Rheum Dis 2015;74:375-80.

29. Svendsen AJ, Junker P, Houen G, Kyvik KO, Nielsen C, Skytthe A, et al. Incidence of chronic persistent rheumatoid arthritis and the impact of smoking: a historical twin cohort study. Arthritis Care Res (Hoboken) 2017;69:616-24.

30. Onozaki K. Etiological and biological aspects of cigarette smoking in rheumatoid arthritis. Inflamm Allergy Drug Targets 2009;8:364-8.

31. Chang K, Yang SM, Kim SH, Han KH, Park SJ, Shin JI. Smoking and rheumatoid arthritis. Int J Mol Sci 2014;15: 22279-95.

32. Kalpakcioglu B, Senel K. The interrelation of glutathione reductase, catalase, glutathione peroxidase, superoxide dismutase, and glucose-6-phosphate in the pathogenesis of rheumatoid arthritis. Clin Rheumatol 2008;27:141-5.

33. Pryor WA, Stone K. Oxidants in cigarette smoke. Radicals, hydrogen peroxide, peroxynitrate, and peroxynitrite. Ann N Y Acad Sci 1993;686:12-27.

34. Sopori M. Effects of cigarette smoke on the immune system. Nat Rev Immunol 2002;2:372-7.

35. Harel-Meir M, Sherer Y, Shoenfeld Y. Tobacco smoking and autoimmune rheumatic diseases. Nat Clin Pract Rheumatol 2007;3:707-15.

36. Benedetti G, Miossec P. Interleukin 17 contributes to the chronicity of inflammatory diseases such as rheumatoid arthritis. Eur J Immunol 2014;44:339-47.
37. Viatte S, Plant D, Raychaudhuri S. Genetics and epigenetics of rheumatoid arthritis. Nat Rev Rheumatol 2013;9:141-53.

38. Zeilinger S, Kühnel B, Klopp N, Baurecht H, Kleinschmidt A, Gieger C, et al. Tobacco smoking leads to extensive genome-wide changes in DNA methylation. PLoS One 2013; 8:e63812.

39. Saevarsdottir S, Rezaei H, Geborek P, Petersson I, Ernestam $\mathrm{S}$, Albertsson $\mathrm{K}$, et al. Current smoking status is a strong predictor of radiographic progression in early rheumatoid arthritis: results from the SWEFOT trial. Ann Rheum Dis 2015;74:1509-14.

40. Anderson R, Meyer PW, Ally MM, Tikly M. Smoking and air pollution as pro-inflammatory triggers for the development of rheumatoid arthritis. Nicotine Tob Res 2016;18:1556-65.

41. Lee YH, Bae SC, Song GG. Gene-environmental interaction between smoking and shared epitope on the development of anti-cyclic citrullinated peptide antibodies in rheumatoid arthritis: a meta-analysis. Int J Rheum Dis 2014;17:528-35.

42. Wagner CA, Sokolove J, Lahey LJ, Bengtsson C, Saevarsdottir $\mathrm{S}$, Alfredsson L, et al. Identification of anticitrullinated protein antibody reactivities in a subset of anti-CCP-negative rheumatoid arthritis: association with cigarette smoking and HLA-DRB1 'shared epitope' alleles. Ann Rheum Dis 2015;74:579-86.

43. Taylor LH, Twigg S, Worthington J, Emery P, Morgan AW, Wilson AG, et al. Metaanalysis of the association of smoking and PTPN22 R620W genotype on autoantibody status and radiological erosions in rheumatoid arthritis. J Rheumatol 2013;40:1048-53.

44. Wu S, Luo H, Xiao X, Zhang H, Li T, Zuo X. Attenuation of collagen induced arthritis via suppression on Th17 response by activating cholinergic anti-inflammatory pathway with nicotine. Eur J Pharmacol 2014;735:97-104.

45. Zhou Y, Zuo X, Li Y, Wang Y, Zhao H, Xiao X. Nicotine inhibits tumor necrosis factor- $\alpha$ induced IL- 6 and IL- 8 secretion in fibroblast-like synoviocytes from patients with rheumatoid arthritis. Rheumatol Int 2012;32:97-104.

46. Vesperini V, Lukas C, Fautrel B, Le Loet X, Rincheval N, Combe B. Association of tobacco exposure and reduction of radiographic progression in early rheumatoid arthritis: results from a French multicenter cohort. Arthritis Care Res (Hoboken) 2013;65:1899-906.

47. Jiang X, Alfredsson L, Klareskog L, Bengtsson C. Smokeless tobacco (moist snuff) use and the risk of developing rheumatoid arthritis: results from a case-control study. Arthritis Care Res (Hoboken) 2014;66:1582-6.

48. Lee J, Luria A, Rhodes C, Raghu H, Lingampalli N, Sharpe O, et al. Nicotine drives neutrophil extracellular traps formation and accelerates collagen-induced arthritis. Rheumatology (Oxford) 2017;56:644-53.

49. de Rooy DP, van Nies JA, Kapetanovic MC, Kristjansdottir $\mathrm{H}$, Andersson ML, Forslind K, et al. Smoking as a risk factor for the radiological severity of rheumatoid arthritis: a study on six cohorts. Ann Rheum Dis 2014;73:1384-7.

50. Söderlin MK, Andersson M, Bergman S; BARFOT study group. Second-hand exposure to tobacco smoke and its effect on disease activity in Swedish rheumatoid arthritis patients. Data from BARFOT, a multicenter study of RA. Clin Exp Rheumatol 2013;31:122-4.

51. Carlens C, Hergens MP, Grunewald J, Ekbom A, Eklund A, Höglund CO, et al. Smoking, use of moist snuff, and risk of 
chronic inflammatory diseases. Am J Respir Crit Care Med 2010;181:1217-22.

52. Maska LB, Sayles HR, O'Dell JR, Curtis JR, Bridges SL Jr, Moreland LW, et al. Serum cotinine as a biomarker of tobacco exposure and the association with treatment response in early rheumatoid arthritis. Arthritis Care Res (Hoboken) 2012;64:1804-10.

53. Andersson ML, Bergman S, Söderlin MK. The effect of stopping smoking on disease activity in rheumatoid arthritis (RA). Data from BARFOT, a multicenter study of early RA. Open Rheumatol J 2012;6:303-9.

54. Fisher MC, Hochberg MC, El-Taha M, Kremer JM, Peng C, Greenberg JD; CORRONA Investigators. Smoking, smoking cessation, and disease activity in a large cohort of patients with rheumatoid arthritis. J Rheumatol 2012;39: 904-9.

55. Di Giuseppe D, Orsini N, Alfredsson L, Askling J, Wolk A. Cigarette smoking and smoking cessation in relation to risk of rheumatoid arthritis in women. Arthritis Res Ther 2013;15:R56.

56. Walsh CA, Conaghan PG. Smoking: a preventable cause of rheumatoid arthritis. Practitioner 2011;255:5.

57. Cuppen BV, Jacobs JW, Ter Borg EJ, Marijnissen AC, Bijlsma JW, Lafeber FP, et al. Necessity of TNF-alpha inhibitor discontinuation in rheumatoid arthritis is predicted by smoking and number of previously used biological DMARDs. Clin Exp Rheumatol 2017;35:221-8.

58. Baka Z, Buzás E, Nagy G. Rheumatoid arthritis and smoking: putting the pieces together. Arthritis Res Ther 2009; 11:238.

59. Naranjo A, Khan NA, Cutolo M, Lee SS, Lazovskis J, Laas K, et al. Smoking cessation advice by rheumatologists: results of an international survey. Rheumatology (Oxford) 2014; 53:1825-9.
60. Naranjo A, Bilbao A, Erausquin C, Ojeda S, Francisco FM, Rúa-Figueroa I, et al. Results of a specific smoking cessation program for patients with arthritis in a rheumatology clinic. Rheumatol Int 2014;34:93-9.

61. Aimer P, Stamp LK, Stebbings S, Cameron V, Kirby S, Croft $S$, et al. Developing a tailored smoking cessation intervention for rheumatoid arthritis patients. Musculoskeletal Care 2016;14:2-14

62. Aimer P, Treharne GJ, Stebbings S, Frampton C, Cameron V, Kirby S, et al. Efficacy of a rheumatoid arthritis-specific smoking cessation program: a randomized controlled pilot trial. Arthritis Care Res (Hoboken) 2017;69:28-37.

63. Aimer P, Stamp LK, Stebbings S, Cameron V, Kirby S, Treharne GJ. Exploring perceptions of a rheumatoid arthritis-specific smoking cessation programme. Musculoskeletal Care 2018;16:74-81.

64. Aimer P, Stamp L, Stebbings S, Valentino N, Cameron V, Treharne GJ. Identifying barriers to smoking cessation in rheumatoid arthritis. Arthritis Care Res (Hoboken) 2015; 67:607-15.

65. Gath ME, Stamp LK, Aimer P, Stebbings S, Treharne GJ. Reconceptualizing motivation for smoking cessation among people with rheumatoid arthritis as incentives and facilitators. Musculoskeletal Care 2018;16:139-46.

66. Roelsgaard IK, Thomsen T, Østergaard M, Christensen R, Hetland ML, Jacobsen S, et al. The effect of an intensive smoking cessation intervention on disease activity in patients with rheumatoid arthritis: study protocol for a randomised controlled trial. Trials 2017; 18:570.

67. Heatherton TF, Kozlowski LT, Frecker RC, Fagerström KO. The Fagerström test for nicotine dependence: a revision of the Fagerström Tolerance Questionnaire. Br J Addict 1991; $86: 1119-27$. 